PROCEEDINGS OF THE

AMERICAN MATHEMATICAL SOCIETY

Volume 27, No. 2, February 1971

\title{
TWO CELLS WITH $N$ POINTS OF LOCAL NONCONVEXITY
}

\author{
NICK M. STAVRAKAS, W. R. HARE AND J. W. KENELLY
}

Abstract. A subset $S$ of the plane is a two cell provided $S$ is homeomorphic to $\{x \mid\|x\| \leqq 1\}$.

THEOREM. Let $S$ be a two cell with exactly $n$ points of local nonconvexity. Then $S$ is expressible as a union of $n+1$ compact convex sets with mutually disjoint interiors.

I. Introduction. We will prove that a two cell $S$ in $R^{2}$ which has exactly $n$ points of local nonconvexity is expressible as a union of $n+1$ compact convex sets with mutually disjoint interiors. It follows immediately that $S$ is $n+1$ polygonally connected, i.e., an $L_{n+1}$ set and thus we have as a corollary a special case of a result of Valentine [1].

Throughout, the symbols $\cup, \cap$ and $\sim$ denote set union, set intersection and set difference respectively. The interior, closure, and boundary of a set $S \subset R^{2}$ are denoted by int $S$, cl $S$ and bd $S$, respectively. The convex hull of a set $S \subset R^{2}$ is denoted by $H(S)$. If $x, y \in S$ then $[x y]$ and $(x y)$ denote the closed and open line segments joining $x$ to $y$, respectively. The Euclidean norm is given by \|\| and $d$ denotes the Hausdorff metric on compact subsets of the plane as given in Valentine [2]. If $S$ is a set, $|S|$ denotes its cardinality. We define the distance between a point $x$ and a set $S$ asinf $\{\|x-y\| \mid y \in S\}$; we denote this distance by $p(x, S)$. By the $\epsilon$ ball about a set $S$ we mean $\{x \mid p(x, S)<\epsilon\}$.

Definition 1. A point $x \in S$ is called a point of local convexity of $S$ if there exists a neighborhood $N$ of $x$ such that $N \cap S$ is convex. If such a neighborhood does not exist, $x$ is called a point of local nonconvexity.

Definition 2. A set $S$ is said to be starshaped relative to a point $p$ if for each $x \in S,[x p] \subset S$.

Definition 3. A segment $[x y]$ is said to be a crosscut of a set $S$ provided $x, y \in$ bd $S$ and $(x y) \subset$ int $S$.

Definition 4. A set $S C R^{2}$ is a two cell provided $S$ is homeomorphic to $\{x \mid\|x\| \leqq 1\}$.

Given a two cell $S$ and a crosscut $[x y]$ of $S,[x y]$ induces a natural decomposition of $S$ into two new two cells $D^{1} x y$ and $D^{2} x y$ such that

Received by the editors February 3, 1970.

A MS 1969 subject classifications. Primary 5225, 5014.

Key words and phrases. Convex set, local convexity, two cell.

Copyright (C) 1971, American Mathematical Society 
$D^{1} x y \cap D^{2} x y=[x y]$. For a proof of this, see Newman [1]. Specifically, the points $x$ and $y$ divide bd $S$ into two disjoint relatively open connected subsets of bd $S$, say $C^{1} x y$ and $C^{2} x y$, and $D^{1} x y$ is the set whose boundary is given by $[x y] \cup C^{1} x y$ and $D^{2} x y$ is the set whose boundary is given by $[x y] \cup C^{2} x y$. If $S$ is a set, $C(S)$ and $L(S)$ will denote its points of local convexity and nonconvexity, respectively, and $\varnothing$ will denote the empty set. The following two theorems constitute the main results of this paper.

THEOREM 1. Let $S \subset R^{2}$ be a two cell such that $|L(S)|=n, n \geqq 2$. Then there exists a crosscut $[x y]$ of $S$ such that $x, y \in C(S)$ and $\left|L\left(D^{i} x y\right)\right|$ $\leqq n-1$ for $i=1,2$.

THEOREM 2. Let $S \subset R^{2}$ be a two cell such that $|L(S)|=n$. Then $S=\bigcup_{i=1}^{n+1} C_{i}$ where $C_{i}, 1 \leqq i \leqq n+1$, are compact, convex and have mutually disjoint interiors.

Theorem 1 is of some independent interest, since it makes induction arguments readily accessible. Also, note that if $n=0$ in Theorem 2 , then the latter reduces to a special case of the important theorem of Tietze [1], which we state for later reference.

TheOREM 3. Let $S$ be a closed connected set in $R^{d}$, all of whose points are points of local convexity. Then $S$ is convex.

We also shall utilize the following result of Valentine [1].

THEOREM 4. Let $S$ be a closed connected set in $R^{d}$ such that $L(S)$ is not empty. Then given $x \in S$, there exists $y \in L(S)$ such that $[x y] \subset S$.

\section{Preliminary results and proof of Theorems 1 and 2.}

THEOREM 5. Let $\left\{A_{i}\right\}_{i=1}^{\infty}$ be a sequence of compact sets in $R^{2}$ converging to a set $A$ in the Hausdorff metric $d$ such that for each $i, A_{i}$ $=\bigcup_{j=1}^{n+1} C_{j}^{t}$ where each $C_{j}^{t}$ is convex and compact for $1 \leqq j \leqq n+1$ and int $C_{j}^{i} \cap$ int $C_{k}^{i}=\varnothing$, for $k \neq j$ and $1 \leqq k, j \leqq n+1$. Then $A=\bigcup_{j=1}^{n+1} C_{j}$ where $C_{j}$ is compact and convex for $1 \leqq j \leqq n+1$ and int $C_{j} \cap$ int $C_{k}=\varnothing$ for $j \neq k, 1 \leqq j, k \leqq n+1$.

Proof. By the theorem of Blaschke we may assume without loss of generality that for each $j$ the sequence $\left\{C_{j}^{i}\right\}_{j=1}^{\infty}$ converges to a set $C_{j}$. Now $C_{j}$ is compact and convex, since $C_{j}$ is the limit of compact convex sets. Since for each $i$ and $j \neq k$ int $C_{j}^{i} \cap$ int $C_{\mathbf{k}}^{i}=\varnothing$, we have int $C_{j}$ กint $C_{k}=\varnothing$, and this completes the proof.

THEOREM 6. Let $S \subset R^{2}$ be a two cell such that $|L(S)|=1$. Then $S=C_{1} \cup C_{2}$ where $C_{1}$ and $C_{2}$ are compact, convex and int $C_{1} \cap$ int $C_{2}=\varnothing$. 
Proof. Throughout the proof let $L(S)=\{x\}$.

Case 1. Suppose there exist $z, q \in$ bd $S$, such that $z \neq x, q \neq x,[z x]$ $\cup[x q] \subset$ bd $S$ and $[z x] \cap[x q]=x$. Then clearly we may choose a crosscut $[x y]$ where $y \in C(S)$ such that $x \in C\left(D^{1} x y\right) \cap C\left(D^{2} x y\right)$. Since $y \in C(S)$, we have $y \in C\left(D^{1} x y\right) \cap C\left(D^{2} x y\right)$. Then $D^{1} x y$ and $D^{2} x y$ are convex by Tietze's Theorem and int $D^{1} x y \cap$ int $D^{2} x y=\varnothing$ by definition. Thus $D^{1} x y$ and $D^{2} x y$ are the required sets.

Case 2. Suppose for each $y \in$ bd $S, y \neq x$, that $[x y] \nsubseteq$ bd $S$. For this case, we need a lemma.

Lemma 1. Let $S$ be a two cell satisfying Case 2. Then if $t \in \mathrm{bd} S, t \neq x$, $(x t)$ Cint $S$.

Proof. Suppose the lemma is false. By Theorem $4, S$ is starshaped relative to $x$, so $[x t] \subset S$. By hypothesis, $(x t) \nsubseteq$ bd $S$, so let $z \in(x t) \cap$ int $S$. Since $S$ is starshaped relative to $x$, for each $m \in(x t) \cap$ int $S$ we have $(x m] C$ int $S$. Then, since we are denying the lemma, we may not find a sequence $\left\{z_{i}\right\}_{i=1}^{\infty}$ such that $z_{i} \in(x t) \cap$ int $S$ such that $\left\{z_{i}\right\}_{i=1}^{\infty}$ converges to $t$, for otherwise $(x t)=\bigcup_{i=1}^{\infty}\left(x z_{i}\right]$ and $\bigcup_{i=1}^{\infty}\left(x z_{i}\right] \subset$ int $S$ by the last sentence, so $(x t) \subset$ int $S$, a contradiction. Thus let $q \neq t$ be the closest point of bd $S \cap[z t]$ to $x$. Then $[t q] \subset$ bd $S$ and $(q x) \subset$ int $S$. Let $q_{1} \in(t q)$. Since $q_{1}$ is a point of local convexity, and since a two cell is the closure of its interior, we may choose $\epsilon>0$, so that $B\left(q_{1}, \epsilon\right) \cap S$ is convex and $D=\operatorname{int}\left(B\left(q_{1}, \epsilon\right) \cap S\right) \neq \varnothing$, where $B\left(q_{1}, \epsilon\right)=\left\{p \mid\left\|p-q_{1}\right\|<\epsilon\right\}$. Let $\mathcal{C}$ $=H(D \cup\{x\}) \sim\{x\}$. Then $\mathcal{C}$ is an open set and $\mathcal{C} C$ int $S$ since $x$ is a seeing point. Now let $\left\{r_{i}\right\}_{i=1}^{\infty}$ be a sequence of boundary points converging to $q$, such that $r_{i} \neq q, r_{i} \notin(t q)$ for each $i$. Then $\left\{r_{i}\right\}_{i=1}^{\infty}$ must approach $q$ from the open half space generated by the line containing $[x t]$, opposite from the open half space containing $\mathcal{C}$. Choose $\delta>0$, so that $E=B(q, \delta) \cap S$ is convex. Then, clearly we have int $(E \cap \mathfrak{e}) \neq \varnothing$. Since $\left\{r_{i}\right\}_{i=1}^{\infty}$ converges to $q$, there exists an integer $k$, such that if $i \geqq k$, we have $r_{i} \in E$. Let $x_{1} \in \operatorname{int}(E \cap \mathfrak{e})$ be such that $q \in\left(r_{k} x_{1}\right) \subset$ int $S$. This contradicts that $q$ is a boundary point.

Returning to Case 2, let $f$ be the homeomorphism mapping the closed unit disc onto $S$. Then bd $S$ is homeomorphic to the unit circle. Let $f\left(x^{*}\right)=x$. Let $y^{*} \neq x^{*}$ be on the unit circle. Then $x^{*}$ and $y^{*}$ naturally divide the unit circle into two nonempty, relatively open, disjoint, connected subsets, say $B_{x^{*} y^{*}}^{1}$ and $B_{x^{*} v^{*}}^{2}$. Let $\left\{x_{i}^{1}\right\}_{i=1}^{\infty}$ and $\left\{x_{i}^{2}\right\}_{i=1}^{\infty}$ be sequences of points in bd $S$ such that the inverse images of $x_{i}^{1}$ and $x_{i}^{2}$ under $f$ are in $B_{x^{*} v^{*}}^{1}$ and $B_{v^{*} x^{*}}^{2}$, respectively, for each $i$, and both sequences coverge to $x$. Now define the set $S_{i}$ for each $i$ to be that subset of $S$ whose boundary is given by $\left[x_{i}^{1} x\right] \cup\left[x x_{i}^{2}\right] \cup f\left(B_{i}\right)$ where $B_{i}$ is $B_{x_{i}}^{1} x_{i}^{2}$ if $x \notin B_{x_{i}}^{1} x_{i}^{2}$ and $B_{i}$ is $B_{x_{i} x_{i}^{2}}^{2}$ if $x \notin B_{x_{i}}^{2} x_{i}^{2}$. Now since $S$ is 
simply connected, $S_{i} \subset S$, and $S_{i}$ for each $i$ is well defined by Lemma 1. Then $\left\{S_{i}\right\}_{i=1}^{\infty}$ converges to $S$ in the Hausdorff metric. Since for each $i, x_{i}^{1}, x_{i}^{2} \in C(S)$, we have $x_{i}^{1}, x_{i}^{2} \in C\left(S_{i}\right)$. Then, the only possible point of local nonconvexity of $S_{i}$ is $x$. For some integer $k$, we must have for each $i \geqq k$, that $x \in L\left(S_{i}\right)$ for otherwise $S$ would be convex. Now by Case 1 each $S_{i}, i \geqq k$, is a union of two compact convex sets with mutually disjoint interiors. Thus, by Theorem $5, S$ is a union of two compact convex sets with mutually disjoint interiors.

Case 3. Suppose there exists $z \in$ bd $S$ such that $z \neq x,[x z] \subset$ bd $S$ and there does not exist $z_{1} \in$ bd $S, z_{1} \neq z$, such that $[x z] \subset\left[x z_{1}\right] \subset$ bd $S$. Further, suppose for each $y \in$ bd $S \sim[x z]$ that $[x y] \nsubseteq \not$ bd $S$. Then, following a similar proof as in Lemma 1 we show that if $y \in$ bd $S \sim[x z]$, then $(x y) C$ int $S$. Finally, as in Case 2 , we construct a sequence of compact sets $\left\{S_{i}\right\}_{i=1}^{\infty}$ such that $\left|L\left(S_{i}\right)\right|=1$ for each $i$ beyond some $k$ and $\left\{S_{i}\right\}_{i=1}^{\infty}$ converges to $S$ in the Hausdorff metric, and then apply Theorem 5.

Proof of Theorem 1. We begin with a lemma.

Lemma 2. Let $S$ be a two cell such that $|L(S)|$ is finite. Let $[x y]$ be a crosscut of $S$ such that $x, y \in C(S)$. Then there exists a convex subset $A x y$ of $S$ such that $(x y) \subset$ int $A x y$.

Proof. Choose $\epsilon>0$ so that $B(x, \epsilon) \cap S$ is convex, $B(y, \epsilon) \cap S$ is convex, $\operatorname{cl} B(x, \epsilon) \cap L(S)=\varnothing$ and $\operatorname{cl} B(y, \epsilon) \cap L(S)=\varnothing$. For each $z \in(x y)$, choose $\epsilon_{z}>0$ so that $\mathrm{cl} B\left(z, \epsilon_{z}\right) \subset$ int $S$. Since [xy] is compact select a finite subcover $\left\{B(x, \epsilon / 2), B(y, \epsilon / 2), B\left(z_{1}, \epsilon z_{1} / 2\right), \cdots\right.$, $\left.B\left(z_{n}, \epsilon z_{n} / 2\right)\right\}$ of the cover $\{B(x, \epsilon / 2), B(y, \epsilon / 2), B(z, \epsilon z / 2) \mid z \in(x y)\}$. Choose $\delta>0$ so that $\delta<\min \left\{\epsilon / 2, \epsilon z_{1} / 2, \cdots, \epsilon z_{n} / 2\right\}$. Let $B$ be the closure of the $\delta / \sqrt{ } 2$ ball about the set $[x y]$. Then $B \cap S$ is clearly connected, closed and $B \cap S \cap L(S)=\varnothing$. Then $B \cap S$ is convex by Tietze's Theorem and this is the required set $A x y$.

Now suppose Theorem 1 is false. Let $r, s \in L(S)$ such that $C_{r s}^{1}$ $\cap L(S)=\varnothing$ or $C_{r s}^{2} \cap L(S)=\varnothing$, which is possible since $|L(S)|$ is finite. Let us suppose that $C_{r s}^{1} \cap L(S)=\varnothing$. Let $\mathfrak{C}=\{[x y] \mid[x y]$ is a crosscut and $x, y \in C(S)\}$. Since $|L(S)|$ is finite, every interior point of $S$ is contained in an element of $\mathfrak{e}$, so int $S \subset \mathrm{C}$. Since we are assuming that Theorem 1 is false, if $[x y] \in \mathcal{e}$, then $x, y \in C_{r z}^{1}$ or $x, y \in C_{r s}^{2}$.

Consider any two crosscuts in $\mathcal{C}$, say $\left[x_{1} y_{1}\right]$ and $\left[x_{2} y_{2}\right]$ such that $x_{1}, y_{1} \in C_{r s}^{1}$ and $x_{2}, y_{2} \in C_{r s}^{2}$. These crosscuts can not intersect. To see this suppose $z \in\left(x_{1} y_{1}\right) \cap\left(x_{2} y_{2}\right)$. Then the set $R$ whose boundary is given by $\left[x_{1} y_{1}\right]$ and the portion $B$ of bd $S$ in $C_{x y}^{1}$ between $x_{1}$ and $y_{1}$, is convex by Tietze's Theorem. Since $B \cap\left[x_{2} y_{2}\right]=\varnothing$, this forces $x_{2}$ or $y_{2}$ 
to be an interior point of $R$, and hence an interior point of $S$, a contradiction.

To continue, the interior of $S$ is connected since it is the homeomorphic image of a connected set. Let $[x y] \in \mathcal{C}$. Let $A x y$ be as in Lemma 2. Then letting $a_{1}=\left\{\right.$ int $\left.A x y \mid[x y] \in \mathcal{C}, x, y \in C_{r s}^{1}\right\}$ and $a_{2}=\left\{\right.$ int $\left.A x y \mid[x y] \in \mathcal{C}, x, y \in C_{r s}^{2}\right\}$, we have int $S \subset \cup a_{1} \cup \cup a_{2}$ since $\cup \mathfrak{e}=U a_{1} \cup \cup a_{2}$. Since int $S$ is connected, we must have $U a_{1} \cap U a_{2}$ $\neq \varnothing$, which says that there exists $\left[x_{1} y_{1}\right]$ and $\left[x_{2} y_{2}\right]$ in $\mathcal{e}$ with $x_{1}$, $y_{1} \in C_{r s}^{1}, x_{2}, y_{2} \in C_{r s}^{2}$ and int $A x_{1} y_{1} \cap$ int $A x_{2} y_{2} \neq \varnothing$. Let $z \in$ int $A x_{1} y_{1}$ nint $A x_{2} y_{2}$. Then $z$ lies in a crosscut having endpoints in $C_{r s}^{1}$ and in a crosscut having endpoints in $C_{r s}^{2}$, which says these crosscuts intersect, which is a contradiction by the last paragraph. Thus Theorem 1 holds.

Proof of Theorem 2. We know Theorem 2 holds when $n=0$, and $n=1$ by Tietze's Theorem and Theorem 6 , respectively. Thus, assume the theorem holds for $0 \leqq k \leqq n-1$ and we will show the result holds for $n$. We begin by considering cases. Let $x \in L(S)$.

Case 1. Suppose there exist $z, q \in \operatorname{bd} S$ such that $z \neq x, q \neq x,[z x]$ $\cup[x q] \subset$ bd $S$ and $[z x] \cap[x q]=\{x\}$. Then choose a crosscut $[x y]$ where $y \in C(S)$ such that $x \in C\left(D^{1} x y\right) \cap C\left(D^{2} x y\right)$. Since $y \in C(S)$, $y \in C\left(D^{1} x y\right) \cap C\left(D^{2} x y\right)$. Now suppose $d=\left|L\left(D^{1} x y\right)\right|$. Then $d \leqq n-1$ and by hypothesis $D^{1} x y=\cup_{i=1}^{d+1} C_{i}$ where for each $i, 1 \leqq i \leqq d+1, C_{i}$ is compact and convex and for $1 \leqq i, j \leqq d+1, i \neq j$, int $C_{i} \cap$ int $C_{j}=\varnothing$. Since $\left|L\left(D_{x y}^{1}\right)\right|=d$ and since $x \in C\left(D^{1} x y\right) \cap C\left(D^{2} x y\right)$, we have $\left|L\left(D^{2} x y\right)\right|$ $=n-d-1$. Thus, by hypothesis $D^{2} x y=\bigcup_{i=1}^{n-d} B_{i}$ where for each $i$, $0 \leqq 1 \leqq n-d, B_{i}$ is compact, convex and for $i \neq j, 1 \leqq i, j \leqq n-d$, int $B_{i}$ nint $B_{j}=\varnothing$. Thus $S$ is a union of $(d+1)+(n-d)=n+1$ compact convex sets with mutually disjoint in teriors.

Case 2. Suppose for each $y \in$ bd $S, y \neq x$, that $[x y] \nsubseteq$ bd $S$. We shall need the following lemma.

Lemma 3. Let $S$ be a two cell such that $|L(S)|=n \geqq 1$ satisfying Case 2 . Let $\left\{x_{i}\right\}_{i=1}^{\infty}$ be a sequence in bd $S$ with $x_{i} \rightarrow x, x \in L(S)$ and $x_{i} \neq x$ for each $i$. Then there exists an integer $k$ such that for each $i \geqq k,\left(x x_{i}\right) \subset \operatorname{int} S$.

Proof. We proceed by induction. We know by Lemma 1 the lemma holds when $|L(S)|=1$. So suppose the lemma holds for $1 \leqq k \leqq n-1$. By Theorem 1, there exists a crosscut $[r s]$ of $S$ such that $r, s \in C(S)$ and $\left|L\left(D_{r s}^{i}\right)\right| \leqq n-1$ for $i=1,2$. Suppose $x \in L\left(D_{r s}^{1}\right)$. Then since there exists an $\epsilon>0$ such that $B(x, \epsilon) \cap S \subset D_{r s}^{1}$, the result holds. The same argument applies if $x \in D_{r s}^{2}$.

To complete Case 2, we argue the same way as in Case 2 of Theorem 6. We construct a sequence of sets $\left\{S_{i}\right\}_{i=1}^{\infty}$ such that $\left\{S_{i}\right\}_{i=1}^{\infty}$ converges to $S$ in the Hausdorff metric and using Case 1 of this theorem 
we show each $S_{i}$ is representable as $n+1$ compact convex sets with mutually disjoint interiors and then apply Theorem 5. This completes Case 2.

Case 3. Suppose there exists $x \in L(S)$ and $z \in b d S, z \neq x$ such that $[z x] \subset$ bd $S$ and there does not exist $z_{1} \in$ bd $S, z_{1} \neq z$ such that $[x z]$ $\subset\left[x z_{1}\right] \subset$ bd $S$. Further suppose for each $y \in$ bd $S \sim[x z]$, that $[x, y]$ $\lceil$ bd $S$. Then using a similar proof as in Lemma 3 , we prove that if $\left\{x_{i}\right\}_{i=1}^{\infty}$ is a sequence in bd $S \sim[x z]$ converging to $x$, then there exists an integer $k$, such that for each $i \geqq k,\left(x_{i} x\right) \subset$ int $S$ and use the same construction as in Case 2 to get the theorem. This completes the proof of Theorem 2.

III. Consequences of Theorem 2. As immediate corollaries of Theorem 2 we have

Corollary 1. Let $S$ be a two cell such that $|L(S)|=n$. Then $S$ is an $L_{n+1}$ set.

Corollary 2. Let $S$ be a two cell such that $|L(S)|=n$. Then for every line $L, L \cap S$ is a union of at most $n+1$ closed line segments.

Corollary 2 is a generalization of the familiar fact that the intersection of a line and a compact convex set is a point or a closed line segment.

Examples are easily constructable to show the number $n+1$ is best in Theorem 2.

\section{REFERENCES}

M. H. A. Newman

1. Elements of the topology of plane sets of points, 2nd ed., Cambridge Univ. Press, Cambridge, 1951, pp. 144-145. MR 13, 483.

H. TIETZE

1. Über Convexheit im kleinen und im grossen und über gewisse den Punkten einer Menge zugeordnete Dimensionzahlen, Math. Z. 28 (1928), 697-707.

F. A. Valentine

1. Local convexity and $L_{n}$ sets, Proc. Amer. Math. Soc. 16 (1965), 1305-1310. MR 32 \#2976.

2. Convex sets, McGraw-Hill Series in Higher Math., McGraw-Hill, New York, 1964. MR $30 \# 503$.

Clemson University, Clemson, South Carolina 29631 